\title{
AS DIFERENTES FASES DO CICLO MENSTRUAL NÃO INFLUENCIAM O RENDIMENTO DE ATLETAS DE NADO SINCRONIZADO
}

\author{
THE DIFFERENT PHASES OF THE MENSTRUAL CYCLE DO NOT INFLUENCE THE PERFORMANCE OF \\ SYNCHRONIZED SWIMMING ATHLETES
}

\section{LAS DIFERENTES FASES DEL CICLOMENSTRUAL NO INFLUENCIAN EL RENDIMIENTO DE ATLETAS DENADO SINCRONIZADO}

\section{Angélica Gregório de Souza (Profissional de Educação Física) Victor Luís dos Santos Passos ${ }^{1}$ (Profissional de Educação Física) Wemson Wangler Ferreira Cardoso (Profissional de Educação Física) Kátia Regina Ponciano (Profissional de Educação Física) Meico Fugita' (Profissional de Educação Física) Aylton José Figueira Junior ${ }^{1}$ (Profissional de Educação Física) Danilo Sales Bocalini² (Profissional de Educação Física)}

1. Laboratório de Fisiologia Translacional, Programa de Pós Graduação em Educação Física, Universidade São Judas Tadeu, São Paulo, Brasil.

2. Centro de Educação Física e Desporto. Universidade Federal do Espirito Santo, Vitoria,

Espirito Santo, Brasil

\section{Correspondência:}

Laboratório de Fisiologia

Translacional da Universidade São Judas Tadeu.

Rua Militão Barbosa de Lima, 132, Centro, São Bernardo do Campo, SP, Brasil. 09720-420.

bocaliniht@hotmail.com

\section{RESUMO}

Introdução: O conhecimento referente à influência das fases do ciclo menstrual no desempenho ainda é inconclusivo. Objetivo: Analisar o desempenho físico e técnico de atletas de nado sincronizado em três diferentes fases do ciclo menstrual. Métodos: Seis atletas de nível nacional (idade: 17,6 $\pm 6,15)$ com ciclo menstrual normal foram avaliadas, considerando as seguintes fases do ciclo menstrual, folicular (F), ovulatória (O) e lútea (L). Foram avaliados os parâmetros antropométricos, neuromotores, o deslocamento vertical, alçada de eggbeater e o desempenho na rotina técnica. Resultados: Não foram encontradas alterações de parâmetros antropométricos, força dos membros superiores $(F ; O ; L)$; abdominal $(F ; O ; L)$ e flexibilidade $(F ; O$; L). Nos testes específicos, tanto deslocamento vertical, alçada de eggbeater $(F ; O ; L)$ e desempenho técnico (F: $77 \pm$ 4, O: $80 \pm$ 5, L: $77 \pm$ 4; pontos) não se alteraram ao longo das fases do ciclo menstrual. Conclusão: Os dados do presente estudo indicam que as fases do ciclo menstrual não exercem influência direta no desempenho de atletas de nado sincronizado.

Descritores: ciclo menstrual; desempenho atlético; esportes/fisiologia; natação.

\section{ABSTRACT}

Introduction: Knowledge regarding the influence of the phases of the menstrual cycle on the performance is still inconclusive. Objective: To analyze the physical and technical performance of synchronized swimmers in three

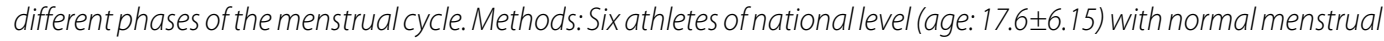
cycle were evaluated considering the following phases of the menstrual cycle, namely, follicular (F), ovulatory (O) and luteal (L). Anthropometric, and neuromotor parameters, vertical displacement, eggbeater kick and performance in the technical routine were evaluated. Results: No changes were found in the anthropometric parameters, strength of the upper limbs (F; $\mathrm{O} ; \mathrm{L})$; abdominal $(F ; O ; L)$ and flexibility $(F ; O ; L)$. In the specific tests, vertical displacement, eggbeater kick (F;O; L), and technical performance (F: $77 \pm 4 ; O: 80 \pm 5 ; L: 77 \pm 4$ points) did not change throughout the menstrual cycle. Conclusion: The data of the present study indicate that the phases of the menstrual cycle do not directly influence the performance of synchronized swimming athletes.

Keywords: menstrual cycle; athletic performance; sports/physiology; swimming.

\section{RESUMEN}

Introducción: El conocimiento referente a la influencia de las fases del ciclo menstrual en el rendimiento sigue siendo inconcluso. Objetivo: Analizar el rendimiento físico y técnico de atletas de nado sincronizado en tres fases diferentes del ciclo menstrual. Métodos: Se evaluaron seis atletas de nivel nacional (edad: 17.6 \pm 6.15$)$ con ciclo menstrual normal, teniendo en cuenta las siguientes fases del ciclo menstrual: folicular (F), ovulatoria (O) y lútea (L). Se evaluaron los parámetros antropométricos, neuromotores, el desplazamiento vertical, el eggbeater ("batidora") y el rendimiento en la rutina técnica. Resultados: No se encontraron cambios en parámetros antropométricos, fuerza de las extremidades superiores ( $(F, O, L)$; abdominal ( $F, O, L)$ y flexibilidad ( $F, O, L)$. En las pruebas específicas, tanto desplazamiento vertical, eggbeater ( $F, O ; L)$ y rendimiento técnico ( $F: 77 \pm 4, O: 80 \pm 5, L: 77 \pm 4$ puntos) no se alteraron a lo largo de las fases del ciclo menstrual. Conclusión: Los datos de este estudio indican que las fases del ciclo menstrual no ejercen ninguna influencia directa sobre el rendimiento de atletas de nado sincronizado.

Descriptores: ciclo menstrual; rendimiento atlético; deportes/fisiología; natación. 


\section{INTRODUÇÃO}

A participação das mulheres em eventos esportivos intensificou o interesse pelos estudos relacionados ao desempenho físico e esportivo'. A realização de esforços físicos produz constantes mudanças hormonais no organismo feminino, promovendo variações nas concentrações dos hormônios estrogênios (E) e de progesterona $(P)$ em relação ao ciclo menstrual normal ${ }^{2}$. Já está demonstrado na literatura que alterações dos hormônios E e P podem promover alterações fisiológicas que podem comprometer a mulher desportista ${ }^{2,3}$. As alterações destes hormônios podem promover alterações emocionais, cognitivos e físicos decorrentes do ciclo menstrual podendo acarretar mudanças no desempenho físico, sobretudo na temperatura corporal e do metabolismo ${ }^{4}$.

Considerando a modalidade esportiva nado sincronizado (NS), sabe-se que traz uma série de demandas fisiológicas e técnicas durante sua prática ${ }^{5}$. Um estudo ${ }^{6}$ de revisão sistemática identificou e discutiu as respostas fisiológicas em diferentes provas da modalidade, provas de e rotinas (coreografias), livres e obrigatórias ${ }^{6}$. Contudo para nosso conhecimento não existem estudos que investigaram a influência das fases do ciclo menstrual em atletas de nado sincronizado (NS).

Desta forma, o objetivo deste estudo foi investigar as alterações no desempenho físico e técnico de atletas de NS de acordo com as fases do ciclo menstrual.

\section{MATERIAIS E MÉTODOS}

Após aprovação pelo comitê de ética de pesquisa, número do parecer 391.548, ano 2013/2016 e a assinatura do Termo de Consentimento Livre e Esclarecido para os responsáveis e Termo de Assentimento para os atletas de acordo com a Declaração de Helsinque número 1996/96 e 251/97, foi aplicado um estudo de campo de natureza transversal com seis atletas saudáveis das categorias juvenil, júnior e sênior de nado sincronizado que participaram voluntariamente deste estudo. Os seguintes critérios de exclusão foram estabelecidos: não atletas de nado sincronizado, menores de 13 anos, hospitalização recente, doença cardiorrespiratória sintomática ou alterações cardiovasculares; comprometimento cognitivo e condições debilitantes, obesidade acentuada, histórico de qualquer distúrbio relacionado ao sistema endócrino, substâncias ergogênicas e anticoncepcionais orais. As análises ${ }^{7}$ foram realizadas considerando as seguintes fases do ciclo menstrual: entre o $1^{\circ}$ e o $3^{\circ} \mathrm{dia}$ após o fluxo menstrual (fase folicular); o segundo, entre o $10^{\circ}$ e o $14^{\circ} \mathrm{dia}$ (fase ovulatória); e o último, entre o $25^{\circ}$ e o $28^{\circ}$ do ciclo (fase lútea).

A composição corporal e parâmetros antropométricos foram avaliados conforme previas publicações do nosso grupo ${ }^{8}$. A massa corporal (MC) foi determinada em balança digital da marca Toledo (modelo 2096PP/2), com capacidade de até $200 \mathrm{~kg}$ e sensibilidade de $50 \mathrm{~g}$.
A estatura (ES) foi mensurada em estadiômetro da marca SANNY (modelo ES2030), fixado a parede e calibrado em escala de $2 \mathrm{~mm}$, sendo a medida realizada com o cursor em ângulo de $90^{\circ}$ em relação à escala, com a voluntária na posição ortostática e pés unidos, calcâneos, região occipital e cinturas pélvica e escapular. A circunferência abdominal e do quadril foram mensuradas com uso de fita métrica com marcação em milímetro. O percentual de gordura foi mensurado utilizando a equação do índice de adiposidade corporal conforme previas publicações ${ }^{9,10}$, sendo: IAC $(\%)=C Q(\mathrm{~cm}) /$ altura $\times$ raiz quadrada da altura $(\mathrm{m})-18$.

O teste utilizado para avaliar a flexibilidade das praticantes foi o Sentar Alcançar - Banco De Wells ${ }^{11}$. Os instrumentos utilizados foram o Flexômetro (caixa de madeira), colchão e folha de protocolo. A voluntária foi posicionada sentada no colchão com os pés totalmente apoiados na caixa. Com os braços estendidos à frente e com uma mão colocada sobre a outra (palmas das mãos para baixo), executou o movimento de flexão de tronco sobre o quadril, empurrando o taco de madeira sobre a caixa que possuía uma fita métrica milimetrada. O procedimento foi realizado três vezes, considerando-se a maior distância atingida.

O teste de flexão abdominal e de braços foi utilizado para avaliar a resistência muscular ${ }^{12,13}$, sendo registrado o número máximo de repetições executadas corretamente ao longo de um minuto.

Para a realização da filmagem, foi utilizado o método de fotogrametria ${ }^{14}$. Resumidamente, três gravações, com uma câmera posicionada em um tripé ajustado a uma altura de $50 \mathrm{~cm}$ do chão e aproximadamente a $65 \mathrm{~cm}$ da linha da água, as atletas realizaram a alçada de eggbeater. Após a coleta de dados, os vídeos gerados foram analisados quadro a quadro no software Kinovea ${ }^{\circledR}$ (Versão 0.8.7, 2010) por três pesquisadores previamente treinados. Na sequência de imagens (Figura 1), foram selecionados os momentos de base, precedente a $A E$, em que o vértex aparece na linha da água; e momento do ápice do movimento, instante de maior altura da AE. Os pontos anatômicos utilizados na determinação da dimensão vertical, nos dois momentos acima citados, foram: i) o vértex; e ii) esterno (região do manúbrio esternal). O vértex foi escolhido como ponto anatômico pelo fato de definir bem a linha da água e o local de início do movimento. O mesmo ponto não foi escolhido para o momento final devido a sua possibilidade de variação através da movimentação da cabeça, optando então pela marcação do esterno como ponto final por ser uma região estática.

Após a captura das imagens durante os testes realizados, as mesmas foram analisadas conjuntamente para determinação da dimensão vertical das AE. As imagens correspondentes com os momentos de base e ápice de cada AE foram transferidas e alinhadas no software Corel DRAW (Corel Draw Graphics Suite, Versão 12.0, Corel Corporation, Canadá). Após a marcação nas imagens dos pontos

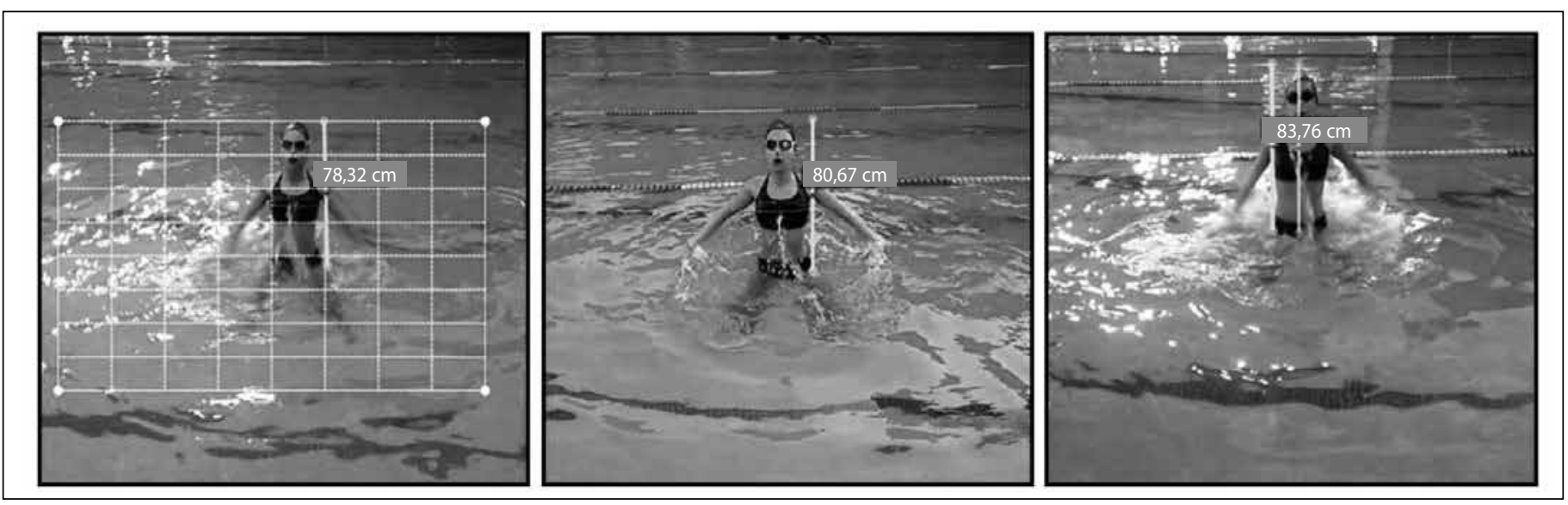

Figura 1. Padrão de média da fotogrametria da alçada de eggbeater. 
supracitados, a altura da AE foi estabelecida com a utilização da ferramenta de medida de deslocamento vertical, sendo considerada a média das três tentativas.

Para a avaliação técnica, foi apresentada uma rotina elaborada pelo grupo de pesquisadores com os seguintes elementos: alçada de tronco, vertical dupla e deslocamento em eggbeater simples e duplo. As filmagens foram realizadas utilizando um Ipad com tela de retina e resolução de 2048 × 1536 pixels, 326 pixels por polegada instalado num tripé de mão e o avaliador acompanhava na lateral da piscina a execução da rotina técnica de cada atleta. Assim os três elementos foram gravados e visualizados como numa competição real, as filmagens foram realizadas por um único avaliador previamente treinado. $O$ critério de notas utilizado foi o contido no manual de regras da FINA assim como os elementos escolhidos ${ }^{15}$. Houve o período de adaptação o qual todas as atletas foram submetidas à quatro tentativas para o processo de familiarização e aquecimento para a realização da rotina. Após este período todas as atletas foram submetidas à realização de quatro tentativas com filmagem dos elementos em sequencial de 1 a 3. Após as filmagens da realização das quatro tentativas, todos os vídeos foram aleatoriamente distribuídos de forma duplo cego e direcionados por e-mail para três juízes de nível A vinculados a Federação Aquática Paulista e Federação Internacional de Natação.

\section{Análise de dados}

Para a análise estatística, foi utilizado o software SPSS para Windows (version 12.0, SPSS Inc., Chicago, Illinois, USA). Os resultados são apresentados como média \pm desvio-padrão. O teste de Kolmogorov-Smirnov foi aplicado para análise da distribuição gaussiana dos dados. Para efeito de comparação o teste ANOVA de medidas repetidas foi aplicado. O nível de significância adotado foi o de $p \leq 0,05$.

\section{RESULTADOS}

Na Tabela 1 é possível visualizar as alterações dos parâmetros antropométricos ao longo das fases do ciclo menstrual das atletas de nado sincronizado. Não foram verificadas diferenças significativas em todos os parâmetros antropométricos nas fases do ciclo menstrual.

De maneira similar aos parâmetros antropométricos, não foi encontrado diferença significativa nos parâmetros neuromotores (Tabela 2) ao longo das fases do ciclo menstrual.

Tabela 1. Parâmetros antropométricos de atletas de nado sincronizado de acordo com a fase do ciclo menstrual.

\begin{tabular}{c|c|c|c}
\hline Parâmetros & $\mathbf{1}^{\circ}$ Fase & $\mathbf{2}^{\circ}$ Fase & $\mathbf{3}^{\circ}$ Fase \\
\hline Massa corporal (kg) & $58 \pm 11$ & $58 \pm 12$ & $58 \pm 11$ \\
\hline Estatura (m) & $1,61 \pm 0,05$ & $1,61 \pm 0,05$ & $1,61 \pm 0,05$ \\
\hline IMC (kg/m2) & $22 \pm 2$ & $22 \pm 3$ & $22 \pm 3$ \\
\hline Massa gorda gordura $(\mathrm{kg})$ & $27 \pm 4$ & $26 \pm 3$ & $27 \pm 3$ \\
\hline Massa livre de gordura (kg) & $15 \pm 4$ & $16 \pm 4$ & $16 \pm 4$ \\
\hline Circunferência abdominal (cm) & $73 \pm 6$ & $74 \pm 7$ & $74 \pm 8$ \\
\hline Circunferência quadril (cm) & $91 \pm 9$ & $92 \pm 9$ & $93 \pm 9$ \\
\hline
\end{tabular}

Valores expressos em média $\pm \mathrm{DP}$.
Não foram encontradas diferenças significativas no deslocamento vertical da alçada de eggbeater, de acordo com a fase do ciclo menstrual, ( $1^{\circ}$ fase: $77 \pm 4,2^{\circ}$ fase: $80 \pm 5,3^{\circ}$ fase: $77 \pm 4 ; \mathrm{cm}$ ) (Figura 2 ).

A Figura 3 apresenta a pontuação atribuída pelos juízes referentes aos elementos alçada de eggbeater (Painel $A$ ), vertical dupla (Painel B) e deslocamento em eggbeater simples e duplo (Painel C) para a avaliação do desempenho técnico de acordo com as fases do ciclo menstrual. Não houve diferença significativa nos elementos: alçada de eggbeater ( $1^{\circ}$ fase: $6,3 \pm 0,9,2^{\circ}$ fase: $6,2 \pm 0,6,3^{\circ}$ fase: $6,3 \pm$ 0,7 ; pontos), vertical dupla ( $1^{\circ}$ fase: $6,2 \pm 0,7,2^{\circ}$ fase: $6,1 \pm 0,6,3^{\circ}$ fase: $6,2 \pm 0,6$; pontos) e deslocamento em eggbeater simples e duplo $\left(1^{\circ}\right.$ fase: $6,0 \pm 0,6,2^{\circ}$ fase: $5,9 \pm 0,5,3^{\circ}$ fase: $5,9 \pm 0,6$; pontos) ao longo das fases do ciclo menstrual.

Tabela 2. Parâmetros neuromotores de acordo com a fase do ciclo menstrual.

\begin{tabular}{c|c|c|c}
\hline Parâmetros & $\mathbf{1}^{\circ}$ Fase & $\mathbf{2}^{\circ}$ Fase & $\mathbf{3}^{\circ}$ Fase \\
\hline Força abdominal (repetições) & $37 \pm 8$ & $34 \pm 7$ & $33 \pm 3$ \\
\hline Flexão de braço (repetições) & $32 \pm 5$ & $36 \pm 6$ & $34 \pm 7$ \\
\hline Flexibilidade (cm) & $18 \pm 3$ & $19 \pm 3$ & $19 \pm 3$ \\
\hline
\end{tabular}

Valores expressos em média $\pm \mathrm{DP}$.
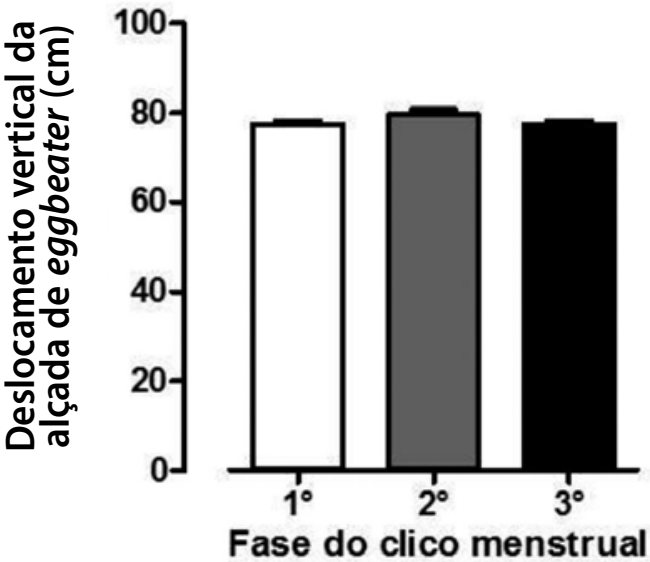

Figura 2. Valores expressos em média \pm desvio padrão do deslocamento vertical da alçada de eggbeater das atletas de nado sincronizado de acordo com a fase do ciclo menstrual.

\section{DISCUSSÃO}

O presente estudo procurou analisar os parâmetros antropométricos, neuromotores e técnicos de atletas de nado sincronizado em três fases do ciclo menstrual: F; O e L não sendo encontradas diferenças tanto nos parâmetros físicos quanto nos técnicos. Adicionalmente, durante o estudo não houve nenhum tipo de complicação por parte ginecológica das atletas e também não foi relatado nenhuma intercorrência clínica no aparelho locomotor que as impossibilitassem de realizar qualquer um dos testes aplicados no estudo.

De acordo com alguns estudos ${ }^{16-19}$ as alterações hormonais podem variar no desempenho físico, porém outros estudos ${ }^{3,4,20}$ salientam que tais alterações não são suficientes para causarem interferências no 
A

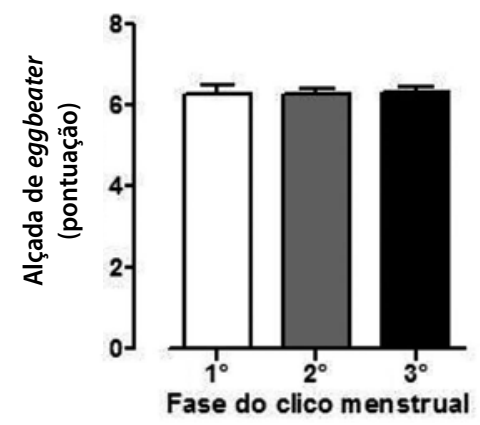

B

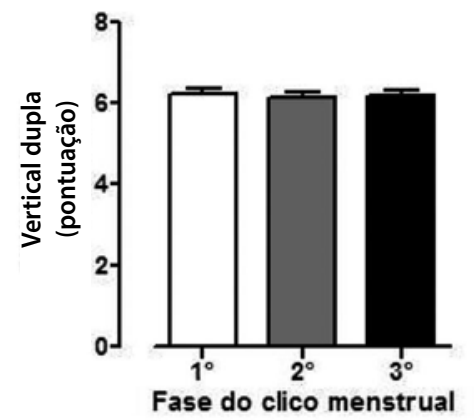

C

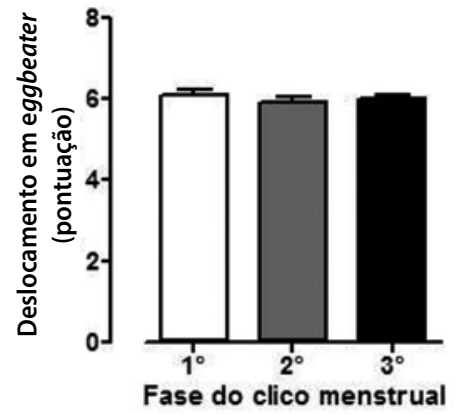

Figura 3. Valores expressos em média \pm desvio padrão dos parâmetros de avaliação técnica das atletas de nado sincronizado de acordo com a fase do ciclo menstrual.

desempenho físico das praticantes. Entretanto, um estudo ${ }^{21}$ relata que o aumento de força no quadríceps durante a metade da fase lútea tem uma relação positiva entre força e concentração de progesterona. De maneira similar ${ }^{22}$ considera que mulheres parecem ser mais fortes na fase lútea, devido ao elevado nível de progesterona em comparação com a fase folicular tardia22. Por outro lado cita que as modificações na mobilidade articular ao longo do ciclo menstrual, sugerindo que na fase lútea a flexibilidade estaria aumentada. Existindo uma possibilidade de que os altos níveis séricos de progesterona aumentam a temperatura corporal na fase lútea, podendo ser responsável por uma maior mobilidade articular ${ }^{4}$

De maneira similar a outros estudos ${ }^{2,23}$ não houve diferenças significativas nos parâmetros antropométricos de acordo com as fases do ciclo menstrual.

Considerando os parâmetros neuromotores, sobretudo a flexibilidade, nossos dados foram similares a outros estudos ${ }^{21,22}$, não sendo encontradas alterações significativas na flexibilidade considerando as fases do ciclo menstrual. Porém, resultados de um estudo ${ }^{17}$ indicaram alterações significativas na flexibilidade, tendo sido atribuído ao aumento da concentração do hormônio relaxina ${ }^{17}$. Sabe-se que este hormônio atua diminuindo a tensão dos tecidos moles e aumenta a lassitude dos tecidos, auxiliando na melhora da flexibilidade; no meio da fase lútea da amostra analisada, tendo seu pico no $14^{\circ}$ dia e no $20^{\circ}$ dia do ciclo menstrual $1^{17}$. Em contraste 22 mesmo tendo realizado controle hormonal não encontrou diferenças significativas na flexibilidade tornando ainda inconsistente a ação deste hormônio em avaliações especificas da flexibilidade, como a analisada no presente estudo e em outros ${ }^{4,21}$. A manutenção da força muscular durante a fase do ciclo menstrual parece ser consenso na literatura ${ }^{7,23}$ embora seja possível encontrar estudos ${ }^{7}$ com alterações significativas na força muscular dos membros inferiores nas fases intermediárias do ciclo menstrual. Por ser uma habilidade de deslocamento e estabilização ${ }^{24}$, a alçada de eggbeater é considerada uma habilidade fundamental do NS ${ }^{24}$ e do polo aquático, exigindo força muscular de membros inferiores ${ }^{25}$. Embora não tenha havido diferenças no deslocamento da alçada de eggbeater que é uma técnica de propulsão vertical, ou seja, de projeção do corpo da atleta acima da superfície da água que pode estar associado ao desempenho de membros inferiores. A altura nesta posição em vertical é um importante indicador de aptidão físico-técnico de atletas de diferentes categorias ${ }^{26}$ sendo um importante componente de avaliação em competições de NS ${ }^{15}$.

O salto vertical é uma das propriedades físicas mais importantes de algumas modalidades como o basquetebol e o voleibol, sendo encontradas diferentes formas de análise desta habilidade ${ }^{27}$. Diferentes parâmetros podem ser medidos através destas ferramentas, por exemplo, a altura máxima alcançada, tempo de voo, etc ${ }^{27}$, contudo, todos os movimentos executados na modalidade nado sincronizado proíbem que os pés toquem no fundo da piscina ${ }^{15}$. Desta forma a alçada de eggbeater executada pelas atletas de nado sincronizado bem como outros movimentos de explosão tentando alcançar máxima altura podem ser um fator interessante de análise, sobretudo considerando a influência tanto de parâmetros físicos ${ }^{25,27}$ quanto técnico ${ }^{27}$. Considerando esta relação físico técnica, os resultados da alçada de eggbeater contraria a tendência de estudos no aumento da força muscular na fase lútea ${ }^{4,7}$, bem como maior mobilidade articular ${ }^{4}$ o que nos permite considerar que tanto a alteração física quanto técnicas podem estar associadas repercutindo no desempenho de ambas as análises.

Algumas limitações neste estudo devem ser consideradas, sendo eles o gap de informações sobre os níveis hormonais durante as diferentes fases do ciclo menstrual impedindo qualquer associação entre os parâmetros avaliados, a falta de testes neuromotores específicos direcionados a modalidade do NS e o número pequeno de atletas avaliadas.

\section{CONCLUSÃO}

Em resumo, os dados do presente estudo indicam que as fases do ciclo menstrual de atletas de nado sincronizado não exercem influência direta no desempenho físico técnico, contudo, estudos com metodologias similares, amostras maiores e uma definição mais precisa das fases do ciclo menstrual para comparações mais detalhadas dos parâmetros avaliados neste estudo.

Todos os autores declararam não haver qualquer potencial conflito de interesses referente a este artigo.

CONTRIBUIÇÕES DOS AUTORES: Cada autor contribuiu individual e significativamente para o desenvolvimento do manuscrito. AGS (0000-0003-4208-7792)*, VLSP $(0000-0002-0114-0713)^{*}$ eWWFC $(0000-0002-5311-316 X)^{*}$ foram os principais contribuintes na coleta de dados. KRP (0000-0002-7274-9606)*, MF (0000-0001-6301-6868)* e DSB (0000-0003-3993-8277)* realizaram a análise estatística e participaram na racionalização do estudo. AGS, VLSP, WWFC e AJFJ (0000-0001-8069-2366)* realizaram a pesquisa bibliográfica. AGS, VLSP, WWFC, KRP, MF, AJFJ e DSB contribuíram com a revisão do manuscrito e com o conceito intelectual do estudo. *ORCID (Open Researcher and Contributor ID). 


\section{REFERÊNCIAS}

1. Rodrigues TR. Força muscular nas fases de fluxo e não fluxo do ciclo menstrual em mulheres praticantes de exercícios resistidos [monografia]. Criciúma: Universidade do Extremo Sul Catarinense; 2010.

2. Lebrun CM. Effect of the different phases of the menstrual cycle and oral contraceptives on athletic performance. Sports Med. 1993;16(6):400-30.

3. Janse de Jonge XA. Effects of the menstrual cycle on exercise performance. Sports Med. 2003;33(11):833-51.

4. Chaves CPG, Simão R, Araújo CGS. Ausência de variação da flexibilidade durante o ciclo menstrual em universitárias. Rev Bras Med Esporte. 2002;8(6):212-8.

5. Mountjoy M. Injuries and medical issues in synchronized Olympic sports. Curr Sports Med Rep. 2009;8(5):255-61.

6. Ponciano K, Miranda ML, Homma M, Miranda JM, Figueira Júnior AJ, Meira Júnior CM, et al. Physiological responses during the practice of synchronized swimming: a systematic review. Clin Physiol Funct Imaging. 2017. doi: 10.1111/cpf.12412.

7. Simão R, Maior AS, Nunes APL, Monteiro L, Chaves CPG. Variações na força muscular de membros superior e inferior nas diferentes fases do ciclo menstrual. R Bras Ci e Mov. 2007;15(3):47-52.

8. Serra AJ, Amaral AM, Rica RL, Barbieri NP, Reis-Júnior D, Silva-Júnior JA, et al. Determinação da densidade corporal por equações generalizadas: facilidade e simplificação no método. ConScientiae Saúde. 2009;8(1):19-24.

9. Bergman RN, Stefanovski D, Buchanan TA, Sumner AE, Reynolds JC, Sebring NG, et al. A better index of body adiposity. Obesity (Silver Spring). 2011;19(5):1083-9.

10. Dias J, Avila M, Damasceno VO, Goncalves R, Barbosa FP, Lamounier JA, et al. Aplicabilidade do indice adiposidade corporal na estimativa do percentual de gordura de jovens mulheres brasileiras. Rev Bras Med Esporte. 2014;20(1):17-20.

11. Wells KF, Dillon EK. The sit and reach - a test of back and leg flexibility. Res Q Exerc Sport. 1952;23:115-8.

12. Matsudo VKR, Matsudo SMM. Avaliação e prescrição da atividade física na criança. Rev Assoc Prof Ed Fís, Londrina. 1995;10:46-55.

13. Astrand PO. From exercise physiology to preventive medicine. Ann Clin Res. 1988;20(1-2):10-7.

14. Guerreiro RC, César EP, Périllier R, Assis CA, Santos TM. Confiabilidade da fotogrametria na medida do deslocamento vertical da alçada de egg no nado sincronizado. R Bras Ci e Mov. 2013;21(3):80-7.

15. Federation Internationale the Natation. FINA synchronised swimming manual for judges, coaches \& referees. Lausanne: FINA; 2013.

16. Foss ML, Keteyian SJ. Bases fisiológicas do exercício e do esporte. 6a. ed. Guanabara Koogan; 2000.

17. Wojtys EM, Huston L, Lindenfeld TN, Hewett TE, Greenfield ML. Association between the menstrua cycle and anterior cruciate ligament injuries in female athletes. Am J Sports Med. 1998;26(5):614-9.

18. Sarwar R, Niclos BB, Rutherford OM. Changes in muscle strength, relaxation rate and fatiguability during the human menstrual cycle. J Physiol. 1996;493(Pt1):267-72.

19. Charkoudian N, Johnson JM. Female reproductive hormones and thermoregulatory control of skin blood flow. Exerc Sport Sci Rev. 2000;28(3):108-12.

20. Hinnerichs KR, Conley DS, Evetovich TK, Engebretsen BJ, Todd JB. Effects of menstrual cycle phase and oral contraceptives on muscular strength, endurance, and flexibility. Med Sci Sports Exerc. 2004;36:S35.

21. Teixeira ALS, Fernandes-Júnior W, Marques FAD, Lacio ML, Dias MRC. Influence of different phases of menstrual cycle on flexibility of young women. Rev Bras Med Esporte. 2012;18(6):361-4.

22. Melegario SM, Simão R, Vale RGS, Batista LA, Novaes JS. A influência do ciclo menstrual na flexibilidade em praticantes de ginástica de academia. Rev Bras Med Esporte. 2006;12(3):125-8.

23. Machado AH, Silva JD, Guanabarino R. Análise da força muscular em mulheres praticantes de musculação na fase menstrual e pós-menstrual. Revista Digital Vida \& Saúde. 2003;2(1):1-9.

24. Fugita M. Fundamentos do nado sincronizado: estabilizar e deslocar. In: Costa PHL. Natação e atividades aquáticas - subsídios para o ensino. São Paulo: Manole; 2010. p. 75.

25. Zinner C, Sperlich B, Krueger M, Focke T, Reed J, Mester J. Strength, Endurance, Throwing Velocity and in-Water Jump Performance of Elite German Water Polo Players. J Hum Kinet. 2015;45:149-56.

26. Marrin $\mathrm{K}$, Theodoros B. Anthropometric and physiological changes in elite female water polo players during a training year. Serb J Sports Sci. 2008;2(1-4):75-83.

27. Sattler T, Sekulic D, Hadzic V, Uljevic O, Dervisevic E. Vertical jumping tests in volleyball: reliability, validity, and playing-position specifics. J Strength Cond Res. 2012;26(6):1532-8. 